\title{
Generating symplectic and Hermitian dual polar spaces over arbitrary fields nonisomorphic to $\mathbb{F}_{2}$
}

\author{
Bart De Bruyn* \\ Department of Pure Mathematics and Computer Algebra \\ Ghent University, Gent, Belgium \\ bdb@cage. ugent. be \\ and \\ Antonio Pasini \\ Dipartimento di Scienze Matematiche e Informatiche \\ Università di Siena, Siena, Italy \\ pasini@unisi.it
}

Submitted: Jan 30, 2007; Accepted: Jul 29, 2007; Published: Aug 4, 2007

Mathematics Subject Classifications: 51A45, 51A50

\begin{abstract}
Cooperstein [6], [7] proved that every finite symplectic dual polar space $D W(2 n-$ $1, q), q \neq 2$, can be generated by $\left(\begin{array}{c}2 n \\ n\end{array}\right)-\left(\begin{array}{c}2 n \\ n-2\end{array}\right)$ points and that every finite Hermitian dual polar space $D H\left(2 n-1, q^{2}\right), q \neq 2$, can be generated by $\left(\begin{array}{c}2 n \\ n\end{array}\right)$ points. In the present paper, we show that these conclusions remain valid for symplectic and Hermitian dual polar spaces over infinite fields. A consequence of this is that every Grassmann-embedding of a symplectic or Hermitian dual polar space is absolutely universal if the (possibly infinite) underlying field has size at least 3 .
\end{abstract}

\section{Introduction}

Let $\Gamma=(P, L, \mathrm{I})$ be a partial linear space, i.e. a rank 2 geometry with point-set $P$, line-set $L$ and incidence relation $\mathrm{I} \subseteq P \times L$ for which every line is incident with at least two points and every two distinct points are incident with at most 1 line. A subspace of $\Gamma$ is a set of points which contains all the points of a line as soon as it contains at least two points of it. If $X$ is a nonempty set of points of $\Gamma$, then $\langle X\rangle_{\Gamma}$ denotes the smallest subspace of $\Gamma$

\footnotetext{
${ }^{*}$ Postdoctoral Fellow of the Research Foundation - Flanders
} 
containing the set $X$. The minimal number $\operatorname{gr}(\Gamma):=\min \left\{|X|: X \subseteq P\right.$ and $\left.\langle X\rangle_{\Gamma}=P\right\}$ of points which are necessary to generate the whole point-set $P$ is called the generating rank of $\Gamma$.

A full embedding $e$ of $\Gamma$ into a projective space $\Sigma$ is an injective mapping $e$ from $P$ to the point-set of $\Sigma$ satisfying: (i) $\langle e(P)\rangle_{\Sigma}=\Sigma$; (ii) $e(L):=\{e(x) \mid x \in L\}$ is a line of $\Sigma$ for every line $L$ of $\Gamma$. The numbers $\operatorname{dim}(\Sigma)$ and $\operatorname{dim}(\Sigma)+1$ are respectively called the projective dimension and the vector dimension of the embedding $e$. The maximal dimension of a vector space $V$ for which $\Gamma$ has a full embedding into $\mathrm{PG}(V)$ is called the embedding rank of $\Gamma$ and is denoted by $\operatorname{er}(\Gamma)$. Certainly, $\operatorname{er}(\Gamma)$ is only defined when $\Gamma$ admits a full embedding, in which case it holds that $\operatorname{er}(\Gamma) \leq \operatorname{gr}(\Gamma)$.

Two embeddings $e_{1}: \Gamma \rightarrow \Sigma_{1}$ and $e_{2}: \Gamma \rightarrow \Sigma_{2}$ of $\Gamma$ are called isomorphic $\left(e_{1} \cong e_{2}\right)$ if there exists an isomorphism $f: \Sigma_{1} \rightarrow \Sigma_{2}$ such that $e_{2}=f \circ e_{1}$. If $e: \Gamma \rightarrow \Sigma$ is a full embedding of $\Gamma$ and if $U$ is a subspace of $\Sigma$ satisfying $(\mathrm{C} 1):\langle U, e(p)\rangle_{\Sigma} \neq U$ for every point $p$ of $\Gamma,(\mathrm{C} 2):\left\langle U, e\left(p_{1}\right)\right\rangle_{\Sigma} \neq\left\langle U, e\left(p_{2}\right)\right\rangle_{\Sigma}$ for any two distinct points $p_{1}$ and $p_{2}$ of $\Gamma$, then there exists a full embedding $e / U$ of $\Gamma$ into the quotient space $\Sigma / U$ mapping each point $p$ of $\Gamma$ to $\langle U, e(p)\rangle_{\Sigma}$. If $e_{1}: \Gamma \rightarrow \Sigma_{1}$ and $e_{2}: \Gamma \rightarrow \Sigma_{2}$ are two full embeddings of $\Gamma$, then we say that $e_{1} \geq e_{2}$ if there exists a subspace $U$ in $\Sigma_{1}$ satisfying $(\mathrm{C} 1),(\mathrm{C} 2)$ and $e_{1} / U \cong e_{2}$. If $e: \Gamma \rightarrow \Sigma$ is a full embedding of $\Gamma$, then by Ronan [17], there exists a unique (up to isomorphism) full embedding $\widetilde{e}: \Gamma \rightarrow \widetilde{\Sigma}$ satisfying (i) $\widetilde{e} \geq e$, (ii) if $e^{\prime} \geq e$ for some full embedding $e^{\prime}$ of $\Gamma$, then $\widetilde{e} \geq e^{\prime}$. We say that $\widetilde{e}$ is universal relative to $e$. If $\widetilde{e} \cong e$ for some full embedding $e$ of $\Gamma$, then we say that $e$ is relatively universal. A full embedding $e$ of $\Gamma$ is called absolutely universal if it is universal relative to any full embedding of $\Gamma$ defined over the same division ring as e. Kasikova and Shult [14] gave sufficient conditions for an embeddable geometry to have an absolutely universal embedding.

The problem of determining generating sets of small size for a given point-line geometry $\Gamma$ is very important for embedding problems. Suppose $X$ is a finite generating set of a geometry $\Gamma$ such that there exists a full embedding $e$ of $\Gamma$ into a projective space $\mathrm{PG}(V)$ with $\operatorname{dim}(V)=|X|$. Then since $|X|=\operatorname{dim}(V) \leq \operatorname{er}(\Gamma) \leq \operatorname{gr}(\Gamma) \leq|X|$, we necessarily have $\operatorname{er}(\Gamma)=\operatorname{gr}(\Gamma)=|X|$. It follows that $e$ is a relatively universal embedding. If moreover the conditions of Kasikova and Shult are satisfied, then we can conclude that $e$ is absolutely universal.

Let $\Pi$ be a non-degenerate polar space of rank $n \geq 2$. With $\Pi$ there is associated a point-line geometry $\Delta$ whose points are the maximal singular subspaces of $\Pi$, whose lines are the next-to-maximal singular subspaces of $\Pi$ and whose incidence relation is reverse containment. We call $\Delta$ a dual polar space (Cameron [4]).

If $x$ and $y$ are two points of $\Delta$, then $\mathrm{d}(x, y)$ denotes the distance between $x$ and $y$ in the point or collinearity graph of $\Delta$. Every convex subspace of $\Delta$ consists of the maximal singular subspaces through a given (possibly empty) singular subspace of $\Pi$. The maximal distance between two points of a convex subspace $A$ of $\Delta$ is called the diameter of $A$. The convex subspaces of diameter 2 , respectively $n-1$, are called the quads, respectively maxes, of $\Delta$. Every dual polar space is an example of a near polygon (Shult and Yanushka [18]; De Bruyn [10]). This means that for every point $x$ and every line $L$, there exists 
a unique point $\pi_{L}(x)$ on $L$ nearest to $x$. More generally, the following property holds in every dual polar space $\Delta$ : if $x$ is a point and $A$ is a convex subspace, then $A$ contains a unique point $\pi_{A}(x)$ nearest to $x$ and $\mathrm{d}(x, y)=\mathrm{d}\left(x, \pi_{A}(x)\right)+\mathrm{d}\left(\pi_{A}(x), y\right)$ for every point $y$ of $A$. We call $\pi_{A}(x)$ the projection of $x$ onto $A$. If $M$ is a max of $\Delta$, then $\mathrm{d}(x, M) \leq 1$ for every point $x$ of $\Delta$.

If $e$ is a full embedding of a thick generalized quadrangle $Q$ into a projective space $\Sigma$, then the underlying division ring of $\Sigma$ is uniquely determined by $Q$ by Tits $[19,8.6]$. In view of the existence of quads in dual polar spaces, a similar conclusion holds for full embeddings of thick dual polar spaces of rank at least 2. By Kasikova and Shult [14, 4.6], every full embedding of a thick dual polar space admits the absolutely universal embedding. By the above we know that the underlying division ring of this absolutely universal embedding space is uniquely determined by $\Delta$; in other words: $\Delta$ admits essentially only one absolutely universal embedding.

In this paper we will determine the generating rank and absolutely universal embedding of all symplectic and Hermitian dual polar spaces whose underlying fields are not isomorphic to the finite field $\mathbb{F}_{2}$ of order 2. Previously, this information was only available in the finite case (see Cooperstein [6], [7]). Several of the lemmas which we will give in this paper are also contained in [6] and [7]. Our intention was to offer the reader a complete and clear discussion of what is known on the generating and embedding ranks of these two families of dual polar spaces. The arguments given in the symplectic and the Hermitian case are very similar, but it has taken us much more effort for the Hermitian dual polar spaces to extend the original results to the infinite case.

We first discuss the symplectic case. Let $V$ be a $2 n$-dimensional vector space $(n \geq 2)$ over a field $\mathbb{K}$ equipped with a non-degenerate alternating form $(\cdot, \cdot)$. Let $\mathrm{PG}(2 n-$ $1, \mathbb{K})$ denote the projective space associated with $V$ and let $\zeta$ denote the symplectic polarity of $\mathrm{PG}(2 n-1, \mathbb{K})$ associated with $(\cdot, \cdot)$. The subspaces of $\mathrm{PG}(2 n-1, \mathbb{K})$ which are totally isotropic with respect to $\zeta$ define a polar space which we denote by $W(2 n-1, \mathbb{K})$. Let $D W(2 n-1, \mathbb{K})$ denote the dual polar space associated with $W(2 n-1, \mathbb{K})$. If $\mathbb{K}$ is isomorphic to the finite field $\mathbb{F}_{q}$ of order $q$, then $W(2 n-1, \mathbb{K})$ and $D W(2 n-1, \mathbb{K})$ are also denoted by $W(2 n-1, q)$ and $D W(2 n-1, q)$.

Let $\bigwedge^{n} V$ denote the $n$-th exterior power of $V$. For every maximal totally isotropic subspace $\alpha=\left\langle\bar{v}_{1}, \bar{v}_{2}, \ldots, \bar{v}_{n}\right\rangle$ of $\mathrm{PG}(2 n-1, \mathbb{K})$, let $e(\alpha)$ be the point $\left\langle\bar{v}_{1} \wedge \bar{v}_{2} \wedge \cdots \wedge\right.$ $\left.\bar{v}_{n}\right\rangle$ of $\mathrm{PG}\left(\bigwedge^{n} V\right)$. Then $e$ defines a full embedding of $D W(2 n-1, \mathbb{K})$ into a subspace of $\operatorname{PG}\left(\bigwedge^{n} V\right)$. This embedding is called the Grassmann-embedding of $D W(2 n-1, \mathbb{K})$. The Grassmann-embedding of $D W(2 n-1, \mathbb{K})$ has vector dimension $\left(\begin{array}{c}2 n \\ n\end{array}\right)-\left(\begin{array}{c}2 n \\ n-2\end{array}\right)$, see for instance Burau [3, 82.7] or De Bruyn [11].

Cooperstein [7] showed that $\operatorname{gr}(D W(2 n-1, q))=\operatorname{er}(D W(2 n-1, q))=\left(\begin{array}{c}2 n \\ n\end{array}\right)-\left(\begin{array}{c}2 n \\ n-2\end{array}\right)$ for any prime power $q \neq 2$. The proof in [7] makes use of some finite group theory, namely some results of Kantor [13]. In the present paper, we give a purely geometrical proof of the above-mentioned result of [7]. This proof does not rely on the finiteness of the underlying field. 
Theorem 1.1 (Section 2) Suppose $n \in \mathbb{N} \backslash\{0,1\}$ and $\mathbb{K}$ is a possibly infinite field not isomorphic to $\mathbb{F}_{2}$. Then there exists a set of $\left(\begin{array}{c}2 n \\ n\end{array}\right)-\left(\begin{array}{c}2 n \\ n-2\end{array}\right)$ points of $D W(2 n-1, \mathbb{K})$ which generate $D W(2 n-1, \mathbb{K})$.

So, if $\mathbb{K} ¥ \mathbb{F}_{2}$ then $\operatorname{er}(D W(2 n-1, \mathbb{K})) \leq \operatorname{gr}(D W(2 n-1, \mathbb{K})) \leq\left(\begin{array}{c}2 n \\ n\end{array}\right)-\left(\begin{array}{c}2 n \\ n-2\end{array}\right)$. Since the Grassmann-embedding of $D W(2 n-1, \mathbb{K})$ has vector-dimension $\left(\begin{array}{c}2 n \\ n\end{array}\right)-\left(\begin{array}{c}2 n \\ n-2\end{array}\right)$, we can conclude

Corollary 1.2 Suppose $n \in \mathbb{N} \backslash\{0,1\}$ and $\mathbb{K}$ is a possibly infinite field not isomorphic to $\mathbb{F}_{2}$. Then

(i) the embedding and generating ranks of $D W(2 n-1, \mathbb{K})$ are equal to $\left(\begin{array}{c}2 n \\ n\end{array}\right)-\left(\begin{array}{c}2 n \\ n-2\end{array}\right)$;

(ii) the Grassmann-embedding of $D W(2 n-1, \mathbb{K})$ is the absolutely universal embedding of $D W(2 n-1, \mathbb{K})$.

Remark. If $\mathbb{K}$ is a field of odd characteristic, then the result of Theorem 1.1 is also covered by a more general result of Blok [1]. The (inductive) proof given in [1] does however not allow to remove the condition on the characteristic of $\mathbb{K}$.

We will now discuss the Hermitian case. Let $\mathbb{K}$ and $\mathbb{K}^{\prime}$ be fields such that $\mathbb{K}^{\prime}$ is a quadratic Galois extension of $\mathbb{K}$, let $\theta$ denote the unique nontrivial element of $\operatorname{Gal}\left(\mathbb{K}^{\prime} / \mathbb{K}\right)$ and let $n \in \mathbb{N} \backslash\{0,1\}$. Let $V$ be a $2 n$-dimensional vector space over $\mathbb{K}^{\prime}$ equipped with a non-degenerate $\theta$-Hermitian form $(\cdot, \cdot)$ of maximal Witt-index $n$. $(\theta$-Hermitian means that $(\bar{w}, \bar{v})=(\bar{v}, \bar{w})^{\theta}$ for all vectors $\bar{v}, \bar{w} \in V$.) Throughout this paper we always assume that a Hermitian form of a vector space is linear in the first argument and semi-linear in the second. Notice that if $\epsilon \in \mathbb{K}^{\prime} \backslash\{0\}$ such that $\epsilon^{\theta}=-\epsilon$ (for instance, $\epsilon=\lambda^{\theta}-\lambda$ for some $\left.\lambda \in \mathbb{K}^{\prime} \backslash \mathbb{K}\right)$, then $(\cdot, \cdot)^{\prime}:=\epsilon \cdot(\cdot, \cdot)$ is a skew- $\theta$-Hermitian form in $V$. Now, let $\mathrm{PG}\left(2 n-1, \mathbb{K}^{\prime}\right)$ denote the projective space associated with $V$ and let $\zeta$ denote the Hermitian polarity of $\operatorname{PG}\left(2 n-1, \mathbb{K}^{\prime}\right)$ associated with the form $(\cdot, \cdot)$. The points of $\operatorname{PG}\left(2 n-1, \mathbb{K}^{\prime}\right)$ which are totally isotropic with respect to $\zeta$ define a $\theta$-Hermitian variety $H\left(2 n-1, \mathbb{K}^{\prime}, \theta\right)$. The subspaces of $\mathrm{PG}\left(2 n-1, \mathbb{K}^{\prime}\right)$ lying on $H\left(2 n-1, \mathbb{K}^{\prime}, \theta\right)$ define a polar space. We denote the associated dual polar space by $D H\left(2 n-1, \mathbb{K}^{\prime}, \theta\right)$. If $\mathbb{K} \cong \mathbb{F}_{q}, \mathbb{K}^{\prime} \cong F_{q^{2}}$ and $\theta: \mathbb{F}_{q^{2}} \rightarrow$ $\mathbb{F}_{q^{2}} ; x \mapsto x^{q}$, then we denote $H\left(2 n-1, \mathbb{K}^{\prime}, \theta\right)$ and $D H\left(2 n-1, \mathbb{K}^{\prime}, \theta\right)$ also by $H\left(2 n-1, q^{2}\right)$ and $D H\left(2 n-1, q^{2}\right)$.

Let $\Lambda^{n} V$ denote the $n$-th exterior power of $V$. For every maximal subspace $\alpha=$ $\left\langle\bar{v}_{1}, \bar{v}_{2}, \ldots, \bar{v}_{n}\right\rangle$ of $H\left(2 n-1, \mathbb{K}^{\prime}, \theta\right)$, let $e(\alpha)$ be the point $\left\langle\bar{v}_{1} \wedge \bar{v}_{2} \wedge \cdots \wedge \bar{v}_{n}\right\rangle$ of $\operatorname{PG}\left(\wedge^{n} V\right)$. By Cooperstein [6] and De Bruyn [12], e defines a full embedding of $D H\left(2 n-1, \mathbb{K}^{\prime}, \theta\right)$ into a Baer- $\mathbb{K}$-subgeometry of $\mathrm{PG}\left(\bigwedge^{n} V\right)$ of dimension $\left(\begin{array}{c}2 n \\ n\end{array}\right)$. This embedding is called the Grassmann-embedding of $D H\left(2 n-1, \mathbb{K}^{\prime}, \theta\right)$.

Cooperstein [6] showed that $\operatorname{gr}\left(D H\left(2 n-1, q^{2}\right)\right)=\operatorname{er}\left(D H\left(2 n-1, q^{2}\right)\right)=\left(\begin{array}{c}2 n \\ n\end{array}\right)$ for any prime power $q \neq 2$. The proof in [6] makes use of some finite group theory, namely some results of Kantor [13]. In the present paper, we give a purely geometrical proof of the above-mentioned result of [6]. This proof does not rely on the finiteness of the underlying field. 
Theorem 1.3 (Section 3) Suppose $n \in \mathbb{N} \backslash\{0,1\}$ and $\mathbb{K} \not \mathbb{F}_{2}$. Then there exists a set of $\left(\begin{array}{c}2 n \\ n\end{array}\right)$ points of $D H\left(2 n-1, \mathbb{K}^{\prime}, \theta\right)$ which generate $D H\left(2 n-1, \mathbb{K}^{\prime}, \theta\right)$.

So, if $\mathbb{K} \approx \mathbb{F}_{2}$ then $\operatorname{er}\left(D H\left(2 n-1, \mathbb{K}^{\prime}, \theta\right)\right) \leq \operatorname{gr}\left(D H\left(2 n-1, \mathbb{K}^{\prime}, \theta\right)\right) \leq\left(\begin{array}{c}2 n \\ n\end{array}\right)$. Since the Grassmann-embedding of $D H\left(2 n-1, \mathbb{K}^{\prime}, \theta\right)$ has vector-dimension $\left(\begin{array}{c}2 n \\ n\end{array}\right)$, we can conclude

Corollary 1.4 Suppose $n \in \mathbb{N} \backslash\{0,1\}$ and $\mathbb{K} \approx \mathbb{F}_{2}$. Then

(i) the embedding and generating ranks of $D H\left(2 n-1, \mathbb{K}^{\prime}, \theta\right)$ are equal to $\left(\begin{array}{c}2 n \\ n\end{array}\right)$;

(ii) the Grassmann-embedding of $D H\left(2 n-1, \mathbb{K}^{\prime}, \theta\right)$ is the absolutely universal embedding of $D H\left(2 n-1, \mathbb{K}^{\prime}, \theta\right)$.

Remarks. (1) The Grassmann-embedding of $D W(2 n-1,2), n \geq 2$, is not absolutely universal. By Blokhuis and Brouwer [2] or $\mathrm{Li}$ [15], the vector dimension of the absolutely universal embedding of $D W(2 n-1,2)$ is equal to $\frac{\left(2^{n}+1\right)\left(2^{n-1}+1\right)}{3}$. For $2 \leq n \leq 5$, the generating rank of $D W(2 n-1,2)$ is also equal to $\frac{\left(2^{n}+1\right)\left(2^{n-1}+1\right)}{3}$ (Cooperstein [5]). The generating rank of $D W(2 n-1,2)$ is unknown for $n \geq 6$.

(2) The Grassmann-embedding of $D H(2 n-1,4), n \geq 3$, is not absolutely universal. By Li [16], the vector dimension of the absolutely universal embedding of $D H(2 n-1,4)$, $n \geq 2$, is equal to $\frac{4^{n}+2}{3}$. For $n \in\{2,3\}$, the generating rank of $D H(2 n-1,4)$ is also equal to $\frac{4^{n}+2}{3}$ (Cooperstein [8]). The generating rank of $D H(2 n-1,4)$ is unknown for $n \geq 4$.

(3) A lot of information on generating and embeddings ranks of point-line geometries (including some of the above geometries) is contained in the survey paper [9].

\section{Proof of Theorem 1.1}

\subsection{Preliminary lemmas}

Let $n \in \mathbb{N} \backslash\{0,1\}$ and let $\mathbb{K}$ be a field. Let $V$ be a $2 n$-dimensional vector space over $\mathbb{K}$ equipped with a non-degenerate alternating form $(\cdot, \cdot)$. Choose a basis $\left\{\bar{e}_{1}, \ldots, \bar{e}_{n}, \bar{f}_{1}, \ldots\right.$, $\left.\bar{f}_{n}\right\}$ in $V$ such that

$$
\left(\bar{e}_{i}, \bar{e}_{j}\right)=\left(\bar{f}_{i}, \bar{f}_{j}\right)=0,\left(\bar{e}_{i}, \bar{f}_{j}\right)=\delta_{i j}
$$

for all $i, j \in\{1, \ldots, n\}$. Here, $\delta_{i j}$ denotes the Kronecker $\delta$ symbol. Let $\mathrm{PG}(2 n-1, \mathbb{K})=$ $\mathrm{PG}(V)$ denote the projective space associated with $V$ and let $\zeta$ denote the symplectic polarity of $\mathrm{PG}(2 n-1, \mathbb{K})$ associated with $(\cdot, \cdot)$. Two points $p_{1}$ and $p_{2}$ of $\mathrm{PG}(2 n-1, \mathbb{K})$ are called orthogonal if $p_{1} \in p_{2}^{\zeta}$. If $p_{1}$ and $p_{2}$ are two non-orthogonal points, then $p_{1} p_{2}$ is called a hyperbolic line. If $\pi$ is a subspace of $\operatorname{PG}(2 n-1, \mathbb{K})$, then the set of all points $p \in \pi$ for which $\pi \subseteq p^{\zeta}$ is called the radical of $\pi$ and is denoted as $\operatorname{Rad}(\pi)$. Obviously, $\operatorname{Rad}(\pi)$ is a subspace of $\pi$. A subspace $\pi$ of $\operatorname{PG}(2 n-1, \mathbb{K})$ is called degenerate if $\operatorname{Rad}(\pi) \neq \emptyset$.

Lemma 2.1 There exist $2 n$ points $p_{1}, p_{2}, \ldots, p_{2 n}$ in $\mathrm{PG}(2 n-1, \mathbb{K})$ such that the following holds for the subspaces $\pi_{i}:=\left\langle p_{1}, p_{2}, \ldots, p_{i}\right\rangle, i \in\{1, \ldots, 2 n\}$ :

(1) for every $i \in\{1, \ldots, n\}$, the subspace $\pi_{2 i}$ is non-degenerate; 
(2) for every $i \in\{1, \ldots, n-1\}, \pi_{2 i+1}$ is degenerate and $\operatorname{Rad}\left(\pi_{2 i+1}\right)$ is a point;

(3) for every $i \in\{2, \ldots, n-1\}, p_{i+1}^{\zeta} \cap \pi_{i}=\pi_{i-1}$;

(4) $\pi_{2 n}=\operatorname{PG}(2 n-1, \mathbb{K})$.

Proof. Put

- $p_{1}=\left\langle\bar{e}_{1}\right\rangle$,

- $p_{2 i}=\left\langle\bar{f}_{i}\right\rangle$ for every $i \in\{1, \ldots, n\}$,

- $p_{2 i+1}=\left\langle\bar{e}_{i}+\bar{e}_{i+1}\right\rangle$ for every $i \in\{1, \ldots, n-1\}$.

Obviously, $\pi_{2 n}=\left\langle\bar{e}_{1}, \ldots, \bar{e}_{n}, \bar{f}_{1}, \ldots, \bar{f}_{n}\right\rangle=\operatorname{PG}(2 n-1, \mathbb{K})$. It is straightforward to verify that $\pi_{2 i}=\left\langle\bar{e}_{1}, \ldots, \bar{e}_{i}, \bar{f}_{1}, \ldots, \bar{f}_{i}\right\rangle, i \in\{1, \ldots, n\}$, is non-degenerate and $\pi_{2 i+1}=$ $\left\langle\bar{e}_{1}, \bar{e}_{2}, \ldots, \bar{e}_{i}, \bar{f}_{1}, \bar{f}_{2}, \ldots, \bar{f}_{i}, \bar{e}_{i+1}\right\rangle, i \in\{1, \ldots, n-1\}$, is degenerate with $\operatorname{Rad}\left(\pi_{2 i+1}\right)=$ $\left\{\left\langle\bar{e}_{i+1}\right\rangle\right\}$.

If $j_{1}, j_{2} \in\{1, \ldots, 2 n\}$ with $j_{1} \leq j_{2}-2$, then clearly $p_{j_{1}} \in p_{j_{2}}^{\zeta}$. If $j \in\{1, \ldots, 2 n-1\}$, then $p_{j} \notin p_{j+1}^{\zeta}$. This proves Claim (3).

Consider now the following point-line incidence structure $\mathcal{N}$ :

- the points of $\mathcal{N}$ are the points of $\mathrm{PG}(2 n-1, \mathbb{K})$;

- the lines of $\mathcal{N}$ are the hyperbolic lines of $\mathrm{PG}(2 n-1, \mathbb{K})$;

- the incidence relation of $\mathcal{N}$ is derived from the one of $\operatorname{PG}(2 n-1, \mathbb{K})$.

Lemma 2.2 Suppose $\mathbb{K} ¥ \mathbb{F}_{2}$ and let $p_{1}, p_{2}, \ldots, p_{2 n}$ be $2 n$ points in $\mathrm{PG}(2 n-1, \mathbb{K})$ satisfying the properties (1) - (4) of Lemma 2.1. Then $\left\langle p_{1}, p_{2}, \ldots, p_{i}\right\rangle_{\mathcal{N}}=\pi_{i} \backslash \operatorname{Rad}\left(\pi_{i}\right)$ for every $i \in\{2, \ldots, 2 n\}$.

Proof. We will prove the lemma by induction on $i$.

If $i=2$, then $\pi_{i}=\pi_{2}=\left\langle p_{1}, p_{2}\right\rangle=\left\langle p_{1}, p_{2}\right\rangle_{\mathcal{N}}$ and $\operatorname{Rad}\left(\pi_{2}\right)=\emptyset$. Suppose therefore that $i \geq 3$ and that the lemma holds for smaller values of $i$.

Suppose $i \geq 3$ is odd and let $p^{*}$ denote the unique point in $\operatorname{Rad}\left(\pi_{i}\right)$. Then $p^{*} \notin$ $\pi_{i-1}$ since $\operatorname{Rad}\left(\pi_{i-1}\right)=\emptyset$. By the induction hypothesis, $\pi_{i-1}=\left\langle p_{1}, p_{2}, \ldots, p_{i-1}\right\rangle_{\mathcal{N}} \subseteq$ $\left\langle p_{1}, p_{2}, \ldots, p_{i}\right\rangle_{\mathcal{N}}$. By considering lines through $p_{i}$, we see that every point of $\pi_{i} \backslash\left(p_{i}^{\zeta} \cap \pi_{i}\right)$ belongs to $\left\langle p_{1}, p_{2}, \ldots, p_{i}\right\rangle_{\mathcal{N}}$. Now, let $p$ be an arbitrary point of $\left(p_{i}^{\zeta} \cap \pi_{i}\right) \backslash\left\{p^{*}\right\}$ and let $L$ denote a line of $\pi_{i}$ through $p$ not contained in $\left(p_{i}^{\zeta} \cap \pi_{i}\right) \cup\left(p^{\zeta} \cap \pi_{i}\right)$. Since $L$ is a hyperbolic line and $L \backslash\{p\} \subseteq\left\langle p_{1}, p_{2}, \ldots, p_{i}\right\rangle_{\mathcal{N}}$, also the point $p$ belongs to $\left\langle p_{1}, p_{2}, \ldots, p_{i}\right\rangle_{\mathcal{N}}$. This proves that $\pi_{i} \backslash \operatorname{Rad}\left(\pi_{i}\right) \subseteq\left\langle p_{1}, p_{2}, \ldots, p_{i}\right\rangle_{\mathcal{N}}$ and hence that $\pi_{i} \backslash \operatorname{Rad}\left(\pi_{i}\right)=\left\langle p_{1}, p_{2}, \ldots, p_{i}\right\rangle_{\mathcal{N}}$.

Suppose $i \geq 4$ is even and let $p^{*}$ denote the unique point in $\operatorname{Rad}\left(\pi_{i-1}\right)$. Since $\operatorname{Rad}\left(\pi_{i-2}\right)=\emptyset, p^{*} \notin \pi_{i-2}$ and hence $p^{*} \notin\left(p_{i}^{\zeta} \cap \pi_{i}\right)$. By the induction hypothesis, $\pi_{i-1} \backslash\left\{p^{*}\right\}=\left\langle p_{1}, p_{2}, \ldots, p_{i-1}\right\rangle_{\mathcal{N}} \subseteq\left\langle p_{1}, \ldots, p_{i}\right\rangle_{\mathcal{N}}$. By considering lines through $p_{i}$, we see that every point of $\pi_{i} \backslash\left(\left(p_{i}^{\zeta} \cap \pi_{i}\right) \cup p_{i} p^{*}\right)$ belongs to $\left\langle p_{1}, p_{2}, \ldots, p_{i}\right\rangle_{\mathcal{N}}$. Now, let $p$ be an arbitrary point of $\left(p_{i}^{\zeta} \cap \pi_{i}\right) \backslash\left\{p_{i}\right\}$ and let $L$ denote a line of $\pi_{i}$ through $p$ not contained in $\left(p^{\zeta} \cap \pi_{i}\right) \cup\left(p_{i}^{\zeta} \cap \pi_{i}\right) \cup\left\langle p, p_{i} p^{*}\right\rangle$. (Notice that if $i=4$, we need the fact that $|\mathbb{K}| \neq 2$ 
for the existence of such a line.) Since $L$ is a hyperbolic line and $L \backslash\{p\} \subseteq\left\langle p_{1}, \ldots, p_{i}\right\rangle_{\mathcal{N}}$, also $p$ belongs to $\left\langle p_{1}, \ldots, p_{i}\right\rangle_{\mathcal{N}}$. This proves that $\pi_{i} \backslash p_{i} p^{*} \subseteq\left\langle p_{1}, p_{2}, \ldots, p_{i}\right\rangle_{\mathcal{N}}$. Now, let $p^{\prime}$ denote an arbitrary point of $p_{i} p^{*}$ and let $L^{\prime}$ denote an arbitrary line of $\pi_{i}$ through $p^{\prime}$ not contained in $\left(p^{\prime \zeta} \cap \pi_{i}\right) \cup p_{i} p^{*}$. Since $L^{\prime}$ is a hyperbolic line and $L^{\prime} \backslash\left\{p^{\prime}\right\} \subseteq\left\langle p_{1}, p_{2}, \ldots, p_{i}\right\rangle_{\mathcal{N}}$, also the point $p^{\prime}$ belongs to $\left\langle p_{1}, \ldots, p_{i}\right\rangle_{\mathcal{N}}$. This proves that $\left\langle p_{1}, \ldots, p_{i}\right\rangle_{\mathcal{N}}=\pi_{i}$.

\section{$2.2 \quad$ A sequence of numbers}

For every $n \in \mathbb{N} \backslash\{0\}$ and every $j \in\{0, \ldots, n\}$, we now define a number $f(n, j)$. For $n=1$, we define

$$
f(1,0)=f(1,1)=1 .
$$

Suppose that for some $n \geq 1$, we have defined $f(n, j)$ for all $j \in\{0, \ldots, n\}$. Then we define

$$
\begin{aligned}
\lambda(n) & :=\sum_{i=0}^{n} f(n, i), \\
f(n+1,0) & :=\lambda(n), \\
f(n+1, j) & :=\sum_{i=j-1}^{n} f(n, i) \text { for every } j \in\{1, \ldots, n+1\} .
\end{aligned}
$$

Notice that $f(n+1,1)=\lambda(n)$. The numbers $f(n, j), \lambda(n)$ were defined in Cooperstein [7]. He also proved the following.

Lemma 2.3 ([7, Proposition 4.3]) Let $n \geq 1$. Then

$$
\begin{aligned}
f(n, 0) & =\left(\begin{array}{c}
2 n-2 \\
n-1
\end{array}\right)-\left(\begin{array}{c}
2 n-2 \\
n-3
\end{array}\right) \\
f(n, j) & =\left(\begin{array}{c}
2 n-1-j \\
n-j
\end{array}\right)-\left(\begin{array}{c}
2 n-1-j \\
n-2-j
\end{array}\right) \text { for every } j \in\{1, \ldots, n\} \\
\lambda(n) & =\left(\begin{array}{c}
2 n \\
n
\end{array}\right)-\left(\begin{array}{c}
2 n \\
n-2
\end{array}\right) .
\end{aligned}
$$

\section{$2.3 \quad$ A generating set of $D W(2 n-1, \mathbb{K}),|\mathbb{K}| \neq 2$}

We keep the notations introduced in Section 2.1. Let $W(2 n-1, \mathbb{K})$ and $\Delta:=D W(2 n-$ $1, \mathbb{K})$ denote the polar and dual polar space associated with the symplectic polarity $\zeta$ of $\mathrm{PG}(2 n-1, \mathbb{K})$. The maximal singular subspaces of $W(2 n-1, \mathbb{K})$ through a given point $x$ of $\mathrm{PG}(2 n-1, \mathbb{K})$ determine a $\max M(x)$ of $\Delta$. The discussion in this subsection is based on Cooperstein [7].

Lemma 2.4 Suppose $x, y$ are non-orthogonal points of $W(2 n-1, \mathbb{K})$ and let $L$ denote the hyperbolic line spanned by $x$ and $y$. Then $\langle M(x), M(y)\rangle_{\Delta}=\bigcup_{z \in L} M(z)$. 
Proof. Let $\alpha$ be an arbitrary point of $M(z), z \in L$. We will show that $\alpha \in\langle M(x)$, $M(y)\rangle_{\Delta}$. Obviously, this holds if $z \in\{x, y\}$. So, suppose $x \neq z \neq y$. Let $\alpha_{x}$ denote the unique maximal singular subspace through $x$ meeting $\alpha$ in an $(n-2)$-dimensional subspace. Then $L \cap\left(\alpha_{x} \cap \alpha\right)=\emptyset$. Since $x, z \in\left(\alpha_{x} \cap \alpha\right)^{\zeta}$, also $y \in\left(\alpha_{x} \cap \alpha\right)^{\zeta}$. Hence, $\alpha_{y}:=\left\langle y, \alpha_{x} \cap \alpha\right\rangle$ is a maximal singular subspace through $y$. Now, $\alpha_{x}, \alpha_{y}$ and $\alpha$ are collinear points of $\Delta$. Since $\alpha_{x} \in M(x)$ and $\alpha_{y} \in M(y), \alpha \in\langle M(x), M(y)\rangle_{\Delta}$.

By the previous paragraph, $\bigcup_{z \in L} M(z) \subseteq\langle M(x), M(y)\rangle_{\Delta}$. Notice that $M(x) \cup M(y) \subseteq$ $\bigcup_{z \in L} M(z)$. So, it remains to show that $\bigcup_{z \in L} M(z)$ is a subspace. Let $\alpha_{1}$ and $\alpha_{2}$ be two distinct maximal singular subspaces of $\bigcup_{z \in L} M(z)$ which are collinear regarded as points of $\Delta$. Then $\operatorname{dim}\left(\alpha_{1} \cap \alpha_{2}\right)=n-2$. Let $\alpha$ denote an arbitrary maximal singular subspace through $\alpha_{1} \cap \alpha_{2}$. Let $x_{1}$ and $x_{2}$ be the unique points of $L$ such that $x_{1} \in \alpha_{1}$ and $x_{2} \in \alpha_{2}$. If $x_{1}=x_{2}$, then $x_{1} \in \alpha$ and hence $\alpha \in M_{x_{1}} \subseteq \bigcup_{z \in L} M(z)$. Suppose $x_{1} \neq x_{2}$. Then $\left(\alpha_{1} \cap \alpha_{2}\right)^{\zeta}=\left\langle\alpha_{1} \cap \alpha_{2}, x_{1}, x_{2}\right\rangle$. So, the maximal singular subspace $\alpha \subseteq\left(\alpha_{1} \cap \alpha_{2}\right)^{\zeta}$ meets $x_{1} x_{2}$ in a point $x_{3} \in L$. Hence, $\alpha \in M_{x_{3}} \subseteq \bigcup_{z \in L} M(z)$.

Lemma 2.5 Suppose $x$ and $y$ are distinct orthogonal points of $W(2 n-1, \mathbb{K})$. Then $\langle M(x), M(y)\rangle_{\Delta}=M(x) \cup M(y)$.

Proof. Clearly, $M(x) \cap M(y)$ is a convex subspace of diameter $n-2$ corresponding with the line $x y$ of $W(2 n-1, \mathbb{K})$.

Let $u \in M(x)$ and $v \in M(y)$ be two distinct collinear points. We show that $u, v \in$ $M(x)$ or $u, v \in M(y)$ (or both). If $u \in M(y)$, then we are done. So, suppose $u \in$ $M(x) \backslash M(y)$. Since $v \in M(y) \cap \Delta_{1}(u), v$ is the unique point of $M(y)$ collinear with $u$. This point coincides with the unique point of $M(x) \cap M(y)$ collinear with $u$. Hence, $v \in M(x)$.

Since $u, v \in M(x)$ or $u, v \in M(y)$, the line $u v$ is contained in $M(x)$ or $M(y)$. It follows that $\langle M(x), M(y)\rangle_{\Delta}=M(x) \cup M(y)$.

Lemma 2.6 Suppose $\mathbb{K}$ is not isomorphic to $\mathbb{F}_{2}$. Let $p_{1}, \ldots, p_{2 n}$ be points of $\mathrm{PG}(2 n-1, \mathbb{K})$ satisfying the properties (1) - (4) of Lemma 2.1. Then $\left\langle M\left(p_{1}\right), M\left(p_{2}\right), \ldots, M\left(p_{n+1}\right)\right\rangle_{\Delta}$ coincides with the whole point set of $\Delta$.

Proof. Put $C=\left\langle p_{1}, \ldots, p_{n+1}\right\rangle$. Then the projective dimension $\operatorname{dim}(C)$ of $C$ is equal to $n$. By Lemmas 2.2, 2.4 and 2.5,

$$
\left\langle M\left(p_{1}\right), M\left(p_{2}\right), \ldots, M\left(p_{n+1}\right)\right\rangle_{\Delta}=\bigcup_{z \in C \backslash \operatorname{Rad}(C)} M(z) .
$$

If $n+1$ is even, then $\operatorname{Rad}(C)=\emptyset$ and hence

$$
\left\langle M\left(p_{1}\right), M\left(p_{2}\right), \ldots, M\left(p_{n+1}\right)\right\rangle_{\Delta}=\bigcup_{z \in C} M(z) .
$$

If $n+1$ is odd, then $\operatorname{Rad}(C)$ is a singleton $\left\{p^{*}\right\}$. If $x \in M\left(p^{*}\right)$, then as both $C$ and $x$ (regarded as subspaces of $\operatorname{PG}(2 n-1, \mathbb{K})$ ) are contained in $p^{* \zeta}, \operatorname{dim}(C \cap x) \geq 1$. It follows 
that $x \in \bigcup_{z \in C \backslash \operatorname{Rad}(C)} M(z)$. So, also if $n+1$ is odd, we have that

$$
\left\langle M\left(p_{1}\right), M\left(p_{2}\right), \ldots, M\left(p_{n+1}\right)\right\rangle_{\Delta}=\bigcup_{z \in C} M(z) .
$$

Now, let $x$ denote an arbitrary point of $\Delta$. As $\operatorname{dim}(x)=n-1$ and $\operatorname{dim}(C)=n, \operatorname{dim}(x \cap$ $C) \geq 0$. Hence, $x \in \bigcup_{z \in C} M(z)=\left\langle M\left(p_{1}\right), M\left(p_{2}\right), \ldots, M\left(p_{n+1}\right)\right\rangle_{\Delta}$. This proves the lemma.

Lemma 2.7 Suppose $\mathbb{K}$ is not isomorphic to $\mathbb{F}_{2}$. Let $p_{1}, p_{2}, \ldots, p_{2 n}$ be 2 n points satisfying the conditions (1) - (4) of Lemma 2.1. Put $B_{0}=\emptyset$ and $B_{j}=\left\langle M\left(p_{1}\right), \ldots, M\left(p_{j}\right)\right\rangle_{\Delta}$ for every $j \in\{1, \ldots, n\}$. Then for every $j \in\{0, \ldots, n\}$, there exists a set $X$ of points in $M\left(p_{j+1}\right)$ satisfying

(i) $|X|=f(n, j)$;

(ii) $\left\langle\left(B_{j} \cap M\left(p_{j+1}\right)\right) \cup X\right\rangle_{\Delta}=M\left(p_{j+1}\right)$.

Proof. We will prove the lemma by induction on $n$.

Suppose $n=2$ and $j \in\{0,1\}$. Then there exists a set $X$ of size $f(2, j)=2$ such that $\langle X\rangle_{\Delta}=M\left(p_{j+1}\right)$. Hence, also $\left\langle\left(B_{j} \cap M\left(p_{j+1}\right)\right) \cup X\right\rangle_{\Delta}=M\left(p_{j+1}\right)$.

Suppose $n=2$ and $j=2$. The point $\left\langle p_{1}, p_{3}\right\rangle$ of $\Delta$ belongs to $B_{2} \cap M\left(p_{3}\right)$. Hence, there exists a set $X$ of size $f(2,2)=1$ such that $\left\langle\left(B_{2} \cap M\left(p_{3}\right)\right) \cup X\right\rangle_{\Delta}=M\left(p_{3}\right)$.

Suppose that $n \geq 3$ and that the lemma holds for smaller values of $n$. By the induction hypothesis and Lemma 2.6, every $M\left(p_{i}\right), i \in\{1, \ldots, 2 n\}$, can be generated by $\lambda(n-1)=$ $\sum_{i=0}^{n-1} f(n-1, i)$ points. As a consequence, the claim holds if $j=0$. So, suppose $j \geq 1$. The singular subspaces through $p_{j+1}$ define a polar space $W(2 n-3, \mathbb{K})$ which lives in the projective space $p_{j+1}^{\zeta} / p_{j+1}$. Let $D W(2 n-3, \mathbb{K})$ denote the dual polar space associated with $W(2 n-3, \mathbb{K})$. There exists a natural bijective correspondence between the points of $D W(2 n-3, \mathbb{K})$ and the points of the $\max M\left(p_{j+1}\right)$. Now, let $\bar{g}_{i}, i \in\{1, \ldots, 2 n\}$, be a nonzero vector of $V$ such that $p_{i}=\left\langle\bar{g}_{i}\right\rangle$. Put

- $p_{i}^{\prime}=p_{i}=\left\langle\bar{g}_{i}\right\rangle$ for every $i \in\{1, \ldots, j-1\}$,

- $p_{j}^{\prime}=\left\langle\left(\bar{g}_{j+1}, \bar{g}_{j+2}\right) \bar{g}_{j}-\left(\bar{g}_{j+1}, \bar{g}_{j}\right) \bar{g}_{j+2}\right\rangle$,

- $p_{i}^{\prime}=p_{i+2}=\left\langle\bar{g}_{i+2}\right\rangle$ for every $i \in\{j+1, \ldots, 2 n-2\}$.

Notice that each of these points belongs to $p_{j+1}^{\zeta}$. Put $p_{i}^{\prime \prime}=p_{i}^{\prime} p_{j+1}$ for every $i \in\{1, \ldots, 2 n-$ $2\}$. Then $p_{i}^{\prime \prime}, i \in\{1, \ldots, 2 n-2\}$, are points of $W(2 n-3, \mathbb{K})$ satisfying the properties $(1)-$ (4) of Lemma 2.1. Let $M^{\prime}\left(p_{i}^{\prime \prime}\right), i \in\{1, \ldots, 2 n-2\}$, denote the max of $M\left(p_{j+1}\right)$ consisting of all maximal singular subspaces through $p_{i}^{\prime} p_{j+1}$. Then $M^{\prime}\left(p_{i}^{\prime \prime}\right)=M\left(p_{i}^{\prime}\right) \cap M\left(p_{j+1}\right)$. The subspace $B_{j} \cap M\left(p_{j+1}\right)$ of $M\left(p_{j+1}\right)$ contains the maxes $M\left(p_{i}\right) \cap M\left(p_{j+1}\right)=M\left(p_{i}^{\prime}\right) \cap$ $M\left(p_{j+1}\right)=M^{\prime}\left(p_{i}^{\prime \prime}\right), i \in\{1, \ldots, j-1\}$. By Lemma 2.6 and the induction hypothesis applied to the maxes $M^{\prime}\left(p_{i}^{\prime \prime}\right), i \in\{j, \ldots, 2 n-2\}$, of $M\left(p_{j+1}\right)$, we see that there exists a set $X$ of size $f(n-1, j-1)+\cdots+f(n-1, n-1)=f(n, j)$ such that $\left\langle\left(B_{j} \cap M\left(p_{j+1}\right)\right) \cup X\right\rangle_{\Delta}=M\left(p_{j+1}\right)$. This proves the lemma.

The following corollary is precisely Theorem 1.1 . 
Corollary 2.8 The dual polar space $D W(2 n-1, \mathbb{K}),|\mathbb{K}| \neq 2$ and $n \geq 2$, can be generated by $\sum_{j=0}^{n} f(n, j)=\lambda(n)=\left(\begin{array}{c}2 n \\ n\end{array}\right)-\left(\begin{array}{c}2 n \\ n-2\end{array}\right)$ points.

Proof. By Lemmas 2.6 and 2.7.

\section{Proof of Theorem 1.3}

\subsection{Some definitions}

Let $\mathbb{K}$ and $\mathbb{K}^{\prime}$ be fields such that $\mathbb{K}^{\prime}$ is a quadratic Galois extension of $\mathbb{K}$, let $\theta$ denote the unique nontrivial element of $\operatorname{Gal}\left(\mathbb{K}^{\prime} / \mathbb{K}\right)$ and let $n \in \mathbb{N} \backslash\{0\}$. Suppose $H$ is a $\theta$-Hermitian variety of $\operatorname{PG}\left(n, \mathbb{K}^{\prime}\right)$. Then one of the following cases occurs for a line $L$ of $\operatorname{PG}\left(n, \mathbb{K}^{\prime}\right)$ :

(1) $L \cap H=\emptyset$;

(2) $|L \cap H|=1$;

(3) $L \subseteq H$

(4) $L \cap H$ is a Baer- $\mathbb{K}$-subline of $L$, i.e., with respect to a suitable reference system of $L$, the points of $L \cap H$ are precisely those points of $L$ whose coordinates can be chosen in the subfield $\mathbb{K}$ of $\mathbb{K}^{\prime}$.

If case (1), (2), (3), respectively (4) occurs, then $L$ is called an exterior line, a tangent line, a totally isotropic line, respectively a secant line.

Let $\mathcal{N}(H)$ denote the point-line incidence structure whose points are the points of $H$, whose lines are the secant lines and whose incidence relation is containment.

\subsection{A useful lemma}

Lemma 3.1 Let $H$ be a non-degenerate Hermitian variety of Witt-index 1 in $\mathrm{PG}\left(2, \mathbb{K}^{\prime}\right)$. Then any three non-collinear points of $\mathcal{N}(H)$ generate the whole point-set of $\mathcal{N}(H)$.

Proof. Let $\theta$ be the involutory automorphism of $\mathbb{K}^{\prime}$ associated with $H$ and let $\mathbb{K}$ denote the fixed field of $\theta$. Let $(\cdot, \cdot)$ denote a skew- $\theta$-Hermitian form of a 3 -dimensional vector space $V$ over $\mathbb{K}^{\prime}$ which gives rise to the Hermitian variety $H$ of $\operatorname{PG}(V)=\operatorname{PG}\left(2, \mathbb{K}^{\prime}\right)$.

Let $p_{1}, p_{2}$ and $p_{3}$ be three mutually distinct points of $\mathcal{N}:=\mathcal{N}(H)$ which are not contained in a line of $\mathcal{N}(H)$. We choose vectors $\bar{e}_{1}, \bar{e}_{2}$ and $\bar{e}_{3}$ in $V$ such that $p_{1}=\left\langle\bar{e}_{1}\right\rangle$, $p_{2}=\left\langle\bar{e}_{2}\right\rangle, p_{3}=\left\langle\bar{e}_{3}\right\rangle,\left(\bar{e}_{1}, \bar{e}_{2}\right)=1$ and $\left(\bar{e}_{1}, \bar{e}_{3}\right)=1$. Put $\lambda:=\left(\bar{e}_{2}, \bar{e}_{3}\right)$. The matrix associated with the skew-Hermitian form $(\cdot, \cdot)$ is equal to

$$
M=\left[\begin{array}{ccc}
0 & 1 & 1 \\
-1 & 0 & \lambda \\
-1 & -\lambda^{\theta} & 0
\end{array}\right] .
$$

The fact that $H$ is nonsingular implies that $\operatorname{det}(M) \neq 0$, or equivalently that $\lambda \notin \mathbb{K}$. So, $\left\{1, \lambda^{\theta}\right\}$ is a basis of $\mathbb{K}^{\prime}$ regarded as two-dimensional vector space over $\mathbb{K}$.

Let $S$ denote the smallest subspace of $\mathcal{N}$ containing the points $p_{1}, p_{2}$ and $p_{3}$. Let $U$ denote the set of points $x$ on $p_{1} p_{2}$ such that $p_{3} x \cap H \subseteq S$. We will show that $\mathrm{U}$ coincides with the whole point-set of $p_{1} p_{2}$. 
Claim I. The points $\left\langle\bar{e}_{1}\right\rangle,\left\langle\bar{e}_{2}\right\rangle$ and $\left\langle\bar{e}_{1}-\frac{1}{\lambda^{\theta+1}} \lambda^{\theta} \bar{e}_{2}\right\rangle$ of $p_{1} p_{2}$ belong to $U$.

Proof. Since $p_{1} p_{3}$ and $p_{2} p_{3}$ are secant lines and $p_{1}, p_{2}, p_{3} \in S,\left\langle\bar{e}_{1}\right\rangle,\left\langle\bar{e}_{2}\right\rangle \in U$. Since $\left(\bar{e}_{1}-\frac{1}{\lambda^{\theta+1}} \lambda^{\theta} \bar{e}_{2}, \bar{e}_{3}\right)=0$, the line $\left\langle\bar{e}_{1}-\frac{1}{\lambda^{\theta+1}} \lambda^{\theta} \bar{e}_{2}, \bar{e}_{3}\right\rangle$ of $\mathrm{PG}\left(2, \mathbb{K}^{\prime}\right)$ intersects $H$ in only the point $p_{3}=\left\langle\bar{e}_{3}\right\rangle$. Hence $\left\langle\bar{e}_{1}-\frac{1}{\lambda^{\theta+1}} \lambda^{\theta} \bar{e}_{2}\right\rangle \in U$.

Claim II. All points $\left\langle\bar{e}_{1}+\left(a+b \lambda^{\theta}\right) \bar{e}_{2}\right\rangle, a, b \in \mathbb{K}$ with $a \neq 0$, belong to $U$.

Proof. A point of $\left(p_{1} p_{3} \cap H\right) \backslash\left\{p_{3}\right\}$ has the form $\left\langle\bar{e}_{1}+k \bar{e}_{3}\right\rangle, k \in \mathbb{K}$. The points of $H \backslash\left\{p_{2}\right\}$ on a line through $\left\langle\bar{e}_{1}+k \bar{e}_{3}\right\rangle, k \in \mathbb{K}$, and $\left\langle\bar{e}_{2}\right\rangle$ are of the form $\left\langle\bar{e}_{1}+k \bar{e}_{3}+\left(k^{\prime}-k k^{\prime} \lambda^{\theta}\right) \bar{e}_{2}\right\rangle, k^{\prime} \in$ $\mathbb{K}$. Hence, for all $k, k^{\prime} \in \mathbb{K},\left\langle\bar{e}_{1}+\left(k^{\prime}-k k^{\prime} \lambda^{\theta}\right) \bar{e}_{2}\right\rangle \in U$. It follows that $\left\langle\bar{e}_{1}+\left(a+b \lambda^{\theta}\right) \bar{e}_{2}\right\rangle \in U$ for all $a, b \in \mathbb{K}$ with $a \neq 0$.

Claim III. Every point $\left\langle\bar{e}_{1}+b \lambda^{\theta} \bar{e}_{2}\right\rangle, b \in \mathbb{K} \backslash\left\{0,-\frac{1}{\lambda^{\theta+1}}\right\}$, belongs to $U$. Proof. Let $b \in \mathbb{K} \backslash\left\{0,-\frac{1}{\lambda^{\theta+1}}\right\}$. Since $\left(\bar{e}_{1}+b \lambda^{\theta} \bar{e}_{2}, \bar{e}_{3}\right)=1+b \lambda^{\theta+1} \neq 0$, the line through $\left\langle\bar{e}_{1}+b \lambda^{\theta} \bar{e}_{2}\right\rangle$ and $p_{3}=\left\langle\bar{e}_{3}\right\rangle$ is a secant line and hence there exists a point of $H$ of the form $\left\langle\bar{e}_{1}+b \lambda^{\theta} \bar{e}_{2}+k^{*} \bar{e}_{3}\right\rangle$. Since $H$ does not contain lines, $\left(\bar{e}_{1}+b \lambda^{\theta} \bar{e}_{2}+k^{*} \bar{e}_{3}, \bar{e}_{2}\right)=1-k^{*} \lambda^{\theta} \neq 0$. The points $\left\langle\bar{e}_{1}+b \lambda^{\theta} \bar{e}_{2}+k^{*} \bar{e}_{3}+k\left(1-k^{*} \lambda^{\theta}\right) \bar{e}_{2}\right\rangle, k \in \mathbb{K}$, all belong to $H$.

If $\left(1-k^{*} \lambda^{\theta}\right)$ is a $\mathbb{K}$-multiple of $\lambda^{\theta}$, then $\left\langle\bar{e}_{1}+k^{*} \bar{e}_{3}\right\rangle \in H$ (so, $\left.k^{*} \in \mathbb{K}\right)$ and $\left\langle\bar{e}_{1}+b \lambda^{\theta} \bar{e}_{2}+\right.$ $\left.k^{*} \bar{e}_{3}\right\rangle$ lies on the line connecting $\left\langle\bar{e}_{1}+k^{*} \bar{e}_{3}\right\rangle \in H$ with $\left\langle\bar{e}_{2}\right\rangle \in H$. By the discussion in the proof of Claim II, $\left\langle\bar{e}_{1}+b \lambda^{\theta} \bar{e}_{2}+k^{*} \bar{e}_{3}\right\rangle=\left\langle\bar{e}_{1}+k \bar{e}_{3}+\left(k^{\prime}-k k^{\prime} \lambda^{\theta}\right) \bar{e}_{2}\right\rangle$ for certain $k, k^{\prime} \in \mathbb{K}$. So, $k^{*}=k, k^{\prime}=0$ and $k k^{\prime}=-b$, which is impossible since $b \neq 0$.

Hence, $1-k^{*} \lambda^{\theta}$ is not a $\mathbb{K}$-multiple of $\lambda^{\theta}$. By Claim II, $\left\langle\bar{e}_{1}+b \lambda^{\theta} \bar{e}_{2}+k^{*} \bar{e}_{3}+\left(1-k^{*} \lambda^{\theta}\right) \bar{e}_{2}\right\rangle \in$ $S$. Since also $\left\langle\bar{e}_{2}\right\rangle \in S$, we have $\left\langle\bar{e}_{1}+b \lambda^{\theta} \bar{e}_{2}+k^{*} \bar{e}_{3}\right\rangle \in S$. It follows that $\left\langle\bar{e}_{1}+b \lambda^{\theta} \bar{e}_{2}\right\rangle \in U$.

By Claims I, II and III, $U$ coincides with the whole point-set of $p_{1} p_{2}$. This implies that $S=H$.

\subsection{On the generation of the geometry $\mathcal{N}(H)$}

Let $\mathbb{K}$ and $\mathbb{K}^{\prime}$ be fields such that $\mathbb{K}^{\prime}$ is a quadratic Galois extension of $\mathbb{K}$, let $\theta$ denote the unique nontrivial element in $\operatorname{Gal}\left(\mathbb{K}^{\prime} / \mathbb{K}\right)$ and let $n \in \mathbb{N} \backslash\{0,1\}$.

Let $V$ be a $2 n$-dimensional vector space over the field $\mathbb{K}^{\prime}$ equipped with a nondegenerate skew- $\theta$-Hermitian form $(\cdot, \cdot)$. Associated with the form $(\cdot, \cdot)$, there is a Hermitian polarity $\zeta$ and a Hermitian variety $H\left(2 n-1, \mathbb{K}^{\prime}, \theta\right)$ of $\mathrm{PG}(V)=\mathrm{PG}\left(2 n-1, \mathbb{K}^{\prime}\right)$. We assume that the Witt-index of $H\left(2 n-1, \mathbb{K}^{\prime}, \theta\right)$ is equal to $n$. Two points $p_{1}$ and $p_{2}$ of $\mathrm{PG}\left(2 n-1, \mathbb{K}^{\prime}\right)$ are called orthogonal if $p_{1} \in p_{2}^{\zeta}$. If $\pi$ is a subspace of $\mathrm{PG}\left(2 n-1, \mathbb{K}^{\prime}\right)$, then the set of all points $p \in \pi$ for which $\pi \subseteq p^{\zeta}$ is called the radical of $\pi$ and is denoted as $\operatorname{Rad}(\pi)$. Obviously, $\operatorname{Rad}(\pi)$ is a subspace of $\pi$. A subspace $\pi$ is called degenerate if $\operatorname{Rad}(\pi) \neq \emptyset$.

Lemma 3.2 There exist points $p_{1}, p_{2}, \ldots, p_{2 n}$ in $H\left(2 n-1, \mathbb{K}^{\prime}, \theta\right)$ satisfying the following properties:

(1) $\pi_{i}:=\left\langle p_{1}, \ldots, p_{i}\right\rangle$ is non-degenerate for every $i \in\{2, \ldots, 2 n\}$;

(2) $\pi_{i} \cap p_{i+1}^{\zeta}=\pi_{i-1}$ for every $i \in\{3, \ldots, 2 n-1\}$;

(3) $\pi_{2 n}=\operatorname{PG}\left(2 n-1, \mathbb{K}^{\prime}\right)$. 
Proof. Choose a basis $\left\{\bar{e}_{1}, \bar{e}_{2}, \ldots, \bar{e}_{n}, \bar{f}_{1}, \bar{f}_{2}, \ldots, \bar{f}_{n}\right\}$ in $V$ such that

$$
\left(\bar{e}_{i}, \bar{e}_{j}\right)=\left(\bar{f}_{i}, \bar{f}_{j}\right)=0,\left(\bar{e}_{i}, \bar{f}_{j}\right)=\delta_{i j}
$$

for all $i, j \in\{1, \ldots, n\}$. Here, $\delta_{i j}$ denotes the Kronecker $\delta$ symbol.

Choose $\mu \in \mathbb{K}^{\prime} \backslash \mathbb{K}$ and put $\lambda_{1}=\frac{\mu^{\theta}}{\mu}$ and $\lambda_{2}=\frac{\mu^{\theta}+1}{\mu+1}$. Then $\lambda_{1} \neq 1 \neq \lambda_{2} \neq \lambda_{1}$. Choose $\lambda \in\left\{\lambda_{1}, \lambda_{2}\right\}$ such that $\lambda \neq-1$. Notice that $\lambda^{\theta+1}=1$ and $\lambda \notin \mathbb{K}$ since $\lambda^{\theta}=\frac{1}{\lambda} \neq \lambda$. Now, put

$$
\begin{aligned}
p_{1} & =\left\langle\bar{e}_{1}\right\rangle, \\
p_{2 k} & =\left\langle\bar{f}_{k}\right\rangle, k \in\{1, \ldots, n\}, \\
p_{2 k+1} & =\left\langle\bar{e}_{k}+\lambda \bar{f}_{k}+\bar{f}_{k+1}+\lambda \bar{e}_{k+1}\right\rangle, k \in\{1, \ldots, n-1\} .
\end{aligned}
$$

Since $\left(\bar{e}_{k}+\lambda \bar{f}_{k}+\bar{f}_{k+1}+\lambda \bar{e}_{k+1}, \bar{e}_{k}+\lambda \bar{f}_{k}+\bar{f}_{k+1}+\lambda \bar{e}_{k+1}\right)=0$, all these points belong to $H\left(2 n-1, \mathbb{K}^{\prime}, \theta\right)$. Obviously, $\pi_{2 n}=\left\langle\bar{e}_{1}, \ldots, \bar{e}_{n}, \bar{f}_{1}, \ldots, \bar{f}_{n}\right\rangle=\mathrm{PG}\left(2 n-1, \mathbb{K}^{\prime}\right)$ and $\pi_{2 i}=$ $\left\langle p_{1}, \ldots, p_{2 i}\right\rangle=\left\langle\bar{e}_{1}, \bar{f}_{1}, \bar{e}_{2}, \bar{f}_{2}, \ldots, \bar{e}_{i}, \bar{f}_{i}\right\rangle$ is non-degenerate for any $i \in\{1, \ldots, n\}$. Suppose that for a certain $i \in\{1, \ldots, n-1\}, \pi_{2 i+1}=\left\langle\bar{e}_{1}, \bar{f}_{1}, \ldots, \bar{e}_{i}, \bar{f}_{i}, \bar{f}_{i+1}+\lambda \bar{e}_{i+1}\right\rangle$ is degenerate. Let $\langle\bar{v}\rangle=\left\langle a_{1} \bar{e}_{1}+b_{1} \bar{f}_{1}+\cdots+a_{i} \bar{e}_{i}+b_{i} \bar{f}_{i}+c\left(\bar{f}_{i+1}+\lambda \bar{e}_{i+1}\right)\right\rangle$ be a point in the radical of $\pi_{2 i+1}$. Since $\langle\bar{v}\rangle$ is orthogonal with $\left\langle\bar{e}_{j}\right\rangle$ and $\left\langle\bar{f}_{j}\right\rangle$, we have $a_{j}=b_{j}=0$ for every $j \in\{1, \ldots, i\}$. So, $\langle\bar{v}\rangle=\left\langle\bar{f}_{i+1}+\lambda \bar{e}_{i+1}\right\rangle$, but this is impossible since $\left(\bar{f}_{i+1}+\lambda \bar{e}_{i+1}, \bar{f}_{i+1}+\lambda \bar{e}_{i+1}\right)=-\lambda^{\theta}+\lambda \neq 0$ (recall $\lambda \notin \mathbb{K})$. We will now prove Claim (2).

Choose a $k \in\{2, \ldots, n\}$ and consider the point $p_{2 k}=\left\langle\bar{f}_{k}\right\rangle$. Obviously, $p_{2 k}=\left\langle\bar{f}_{k}\right\rangle$ and $p_{2 k-1}=\left\langle\bar{e}_{k-1}+\lambda \bar{f}_{k-1}+\bar{f}_{k}+\lambda \bar{e}_{k}\right\rangle$ are not orthogonal. It is also obvious that $p_{2 k}$ and $p_{i}$ are orthogonal for every $i \in\{1, \ldots, 2 k-2\}$.

Choose a $k \in\{2, \ldots, n-1\}$ and consider the point $p_{2 k+1}=\left\langle\bar{e}_{k}+\lambda \bar{f}_{k}+\bar{f}_{k+1}+\lambda \bar{e}_{k+1}\right\rangle$. Obviously, $p_{2 k+1}=\left\langle\bar{e}_{k}+\lambda \bar{f}_{k}+\bar{f}_{k+1}+\lambda \bar{e}_{k+1}\right\rangle$ and $p_{2 k}=\left\langle\bar{f}_{k}\right\rangle$ are not orthogonal. It is also clear that $p_{2 k+1}$ and $p_{i}$ are orthogonal for every $i \in\{1, \ldots, 2 k-2\}$. Since $\left(\bar{e}_{k-1}+\lambda \bar{f}_{k-1}+\right.$ $\left.\bar{f}_{k}+\lambda \bar{e}_{k}, \bar{e}_{k}+\lambda \bar{f}_{k}+\bar{f}_{k+1}+\lambda \bar{e}_{k+1}\right)=-1+\lambda^{\theta+1}=0$, also $p_{2 k+1}$ and $p_{2 k-1}$ are orthogonal.

Put $\mathcal{N}:=\mathcal{N}\left(H\left(2 n-1, \mathbb{K}^{\prime}, \theta\right)\right)$.

Lemma 3.3 Suppose $\mathbb{K} ¥ \mathbb{F}_{2}$ and let $p_{1}, p_{2}, \ldots, p_{2 n}$ be $2 n$ points of $H\left(2 n-1, \mathbb{K}^{\prime}, \theta\right)$ satisfying the properties (1), (2) and (3) of Lemma 3.2. Then $\left\langle p_{1}, p_{2}, \ldots, p_{i}\right\rangle_{\mathcal{N}}=\pi_{i} \cap$ $H\left(2 n-1, \mathbb{K}^{\prime}, \theta\right)$ for every $i \in\{1, \ldots, 2 n\}$.

Proof. We will prove the lemma by induction on $i$. Obviously, the lemma holds if $i \leq 2$. If $i=3$, then the lemma holds by Lemma 3.1. Suppose therefore that $i \geq 4$ and that the lemma holds for smaller values of $i$. By the induction hypothesis, $\pi_{i-1} \cap H\left(2 n-1, \mathbb{K}^{\prime}, \theta\right)=$ $\left\langle p_{1}, \ldots, p_{i-1}\right\rangle_{\mathcal{N}} \subseteq\left\langle p_{1}, \ldots, p_{i}\right\rangle_{\mathcal{N}}$.

(I) Let $p$ denote an arbitrary point of $\pi_{i} \cap H\left(2 n-1, \mathbb{K}^{\prime}, \theta\right)$ not contained in $p_{i}^{\zeta}$. If $p_{i} p$ intersects $\pi_{i-1}$ in a point of $H\left(2 n-1, \mathbb{K}^{\prime}, \theta\right)$ (and hence also of $\left\langle p_{1}, p_{2}, \ldots, p_{i}\right\rangle_{\mathcal{N}}$ ), then $p \in\left\langle p_{1}, \ldots, p_{i}\right\rangle_{\mathcal{N}}$. Suppose therefore that $p_{i} p$ intersects $\pi_{i-1}$ in a point $p^{\prime}$ not belonging to $H\left(2 n-1, \mathbb{K}^{\prime}, \theta\right)$. 
Claim. There exists a secant line $L \subseteq \pi_{i-1}$ through $p^{\prime}$ which intersects $\pi_{i-2}$ in a point not belonging to $H\left(2 n-1, \mathbb{K}^{\prime}, \theta\right)$.

PROOF. Notice first that the tangent lines of $\pi_{i-1}$ through $p^{\prime}$ are precisely those lines through $p^{\prime}$ which contain a point of $\left(p^{\prime \zeta} \cap \pi_{i-1}\right) \cap H\left(2 n-1, \mathbb{K}^{\prime}, \theta\right)$.

The points of $H\left(2 n-1, \mathbb{K}^{\prime}, \theta\right) \cap \pi_{i-1}$ generate $\pi_{i-1}$. Hence, there exists a line $M \subseteq \pi_{i-1}$ through $p^{\prime}$ containing a point of $H\left(2 n-1, \mathbb{K}^{\prime}, \theta\right) \backslash\left(p^{\prime \zeta} \cap \pi_{i-1}\right)$. If the unique point in $M \cap\left(p^{\prime \zeta} \cap \pi_{i-1}\right)$ is contained in $H\left(2 n-1, \mathbb{K}^{\prime}, \theta\right)$, then $M \cap H\left(2 n-1, \mathbb{K}^{\prime}, \theta\right)$ contains at least two points, a contradiction, since every line through $p^{\prime}$ containing a point of $\left(p^{\prime \zeta} \cap \pi_{i-1}\right) \cap H\left(2 n-1, \mathbb{K}^{\prime}, \theta\right)$ is a tangent line. Hence,

$$
M \cap\left(p^{\prime \zeta} \cap \pi_{i-1}\right) \cap H\left(2 n-1, \mathbb{K}^{\prime}, \theta\right)=\emptyset .
$$

By (1), $M$ is not a tangent line. So, $M$ is a secant line since $M \cap H\left(2 n-1, \mathbb{K}^{\prime}, \theta\right) \neq \emptyset$. If the unique point in $M \cap \pi_{i-2}$ is not contained in $H\left(2 n-1, \mathbb{K}^{\prime}, \theta\right)$, then we are done (take $L=M)$. So, suppose $M \cap \pi_{i-2} \subseteq H\left(2 n-1, \mathbb{K}^{\prime}, \theta\right)$. Since $\pi_{i-2}$ is non-degenerate, there exists a secant line $M_{1} \subseteq \pi_{i-2}$ through $M \cap \pi_{i-2}$. Let $\alpha$ be the plane $\left\langle M, M_{1}\right\rangle$ and put $M_{2}:=\alpha \cap\left(p^{\prime \zeta} \cap \pi_{i-1}\right)$. By (1), $M \cap M_{2} \not \subset H\left(2 n-1, \mathbb{K}^{\prime}, \theta\right)$. So, $M_{2}$ cannot be contained in $H\left(2 n-1, \mathbb{K}^{\prime}, \theta\right)$. Obviously, $\operatorname{Rad}(\alpha) \subseteq \alpha \cap\left(p^{\prime \zeta} \cap \pi_{i-1}\right)=M_{2}$. One readily sees that $\alpha$ is degenerate if and only if $M_{2} \cap H\left(2 n-1, \mathbb{K}^{\prime}, \theta\right)$ is a singleton (the radical of $\alpha$ ).

Suppose $\alpha$ is degenerate and let $x^{*}$ denote the unique point in $M_{2} \cap H\left(2 n-1, \mathbb{K}^{\prime}, \theta\right)$. Then any line of $\alpha$ through $p^{\prime}$ not containing $x^{*}$ is a secant line. Now, the lines of $\alpha$ through $p^{\prime}$ intersecting $M_{1} \cap H\left(2 n-1, \mathbb{K}^{\prime}, \theta\right)$ non-trivially define a Baer- $\mathbb{K}$-subline of the quotient space $\alpha / p^{\prime}$ (which is a projective line over $\mathbb{K}^{\prime}$ ). The line $p^{\prime} x^{*}$ defines a point of $\alpha / p^{\prime}$. It follows that there exists a line $L \subseteq \alpha$ through $p^{\prime}$ different from $p^{\prime} x^{*}$ intersecting $M_{1}$ in a point not belonging to $H\left(2 n-1, \mathbb{K}^{\prime}, \theta\right)$. This line $L$ satisfies the required conditions.

Suppose $\alpha$ is non-degenerate and $M_{2} \cap H\left(2 n-1, \mathbb{K}^{\prime}, \theta\right)=\emptyset$. Let $\mathcal{L}_{p^{\prime}}$ denote the set of lines of $\alpha$ through $p^{\prime}$ intersecting $M_{1}$ in a point of $H\left(2 n-1, \mathbb{K}^{\prime}, \theta\right)$. If there exists a line $L \notin \mathcal{L}_{p^{\prime}}$ in $\alpha$ through $p^{\prime}$ containing a point of $H\left(2 n-1, \mathbb{K}^{\prime}, \theta\right)$, then this line $L$ satisfies all required conditions. Suppose therefore that all points of $\alpha \cap H\left(2 n-1, \mathbb{K}^{\prime}, \theta\right)$ are contained in a line of $\mathcal{L}_{p^{\prime}}$. Let $L_{1}$ and $L_{2}$ denote two distinct lines of $\mathcal{L}_{p^{\prime}}$. Put $\left\{u_{1}\right\}=L_{1} \cap M_{1}$ and let $u_{2}$ denote the unique point of $L_{2}$ such that $u_{1} u_{2}$ is a tangent line. Let $x$ denote an arbitrary point of $L_{2} \backslash\left\{p^{\prime}, u_{2}\right\}$. Then the line $u_{1} x$ is a secant line. Hence, the set of lines through $p^{\prime}$ meeting $u_{1} x$ in a point of $H\left(2 n-1, \mathbb{K}^{\prime}, \theta\right)$ is a Baer- $\mathbb{K}$-subline of the quotient space $\alpha / p^{\prime}$ which necessarily coincides with $\mathcal{L}_{p^{\prime}}$. It follows that $x \in H\left(2 n-1, \mathbb{K}^{\prime}, \theta\right)$. Since $x$ was an arbitrary point of $L_{2} \backslash\left\{p^{\prime}, u_{2}\right\}, L_{2} \backslash\left\{p^{\prime}, u_{2}\right\}$ is contained in $H\left(2 n-1, \mathbb{K}^{\prime}, \theta\right)$. Since $\left|\mathbb{K}^{\prime} \backslash \mathbb{K}\right|>2\left(\right.$ recall $\left.\mathbb{K} \neq \mathbb{F}_{2}\right)$, this implies that $L_{2} \subseteq H\left(2 n-1, \mathbb{K}^{\prime}, \theta\right)$, in contradiction with $p^{\prime} \notin H\left(2 n-1, \mathbb{K}^{\prime}, \theta\right)$.

Suppose $\alpha$ is non-degenerate and $M_{2} \cap H\left(2 n-1, \mathbb{K}^{\prime}, \theta\right)$ is a Baer- $\mathbb{K}$-subline of $M_{2}$. Let $x_{i}, i \in\{1,2\}$, denote an arbitrary point of $\left(M_{i} \cap H\left(2 n-1, \mathbb{K}^{\prime}, \theta\right)\right) \backslash M_{3-i}$. Then $x_{1} x_{2} \cap H\left(2 n-1, \mathbb{K}^{\prime}, \theta\right)$ is a Baer- $\mathbb{K}$-subline of $x_{1} x_{2}$. So, $p^{\prime} \notin x_{1} x_{2}$. (Recall that lines through $p^{\prime}$ containing a point of $M_{2} \cap H\left(2 n-1, \mathbb{K}^{\prime}, \theta\right)$ are tangent lines.) If $u \in x_{1} x_{2} \cap H(2 n-$ $\left.1, \mathbb{K}^{\prime}, \theta\right) \backslash\left\{x_{2}\right\}$, then the line $p^{\prime} u$ is a secant line and intersects $M_{2}$ in a point not belonging to $H\left(2 n-1, \mathbb{K}^{\prime}, \theta\right)$. If $p^{\prime} u$ intersects $M_{1}$ in a point not belonging to $H\left(2 n-1, \mathbb{K}^{\prime}, \theta\right)$, then we are done (take $L=p^{\prime} u$ ). So, suppose that all lines $p^{\prime} u, u \in x_{1} x_{2} \cap H\left(2 n-1, \mathbb{K}^{\prime}, \theta\right) \backslash\left\{x_{2}\right\}$ 
intersect $M_{1}$ in a point of $H\left(2 n-1, \mathbb{K}^{\prime}, \theta\right)$. Let $\mathcal{L}_{1}$ denote the set of lines through $p^{\prime}$ intersecting $M_{1}$ in a point of $H\left(2 n-1, \mathbb{K}^{\prime}, \theta\right)$ and let $\mathcal{L}_{2}$ denote the set of lines through $p^{\prime}$ intersecting $x_{1} x_{2}$ in a point of $H\left(2 n-1, \mathbb{K}^{\prime}, \theta\right)$. Then $\mathcal{L}_{1}$ and $\mathcal{L}_{2}$ are two Baer- $\mathbb{K}$-sublines of $\alpha / p^{\prime}$. Since $\left|\mathcal{L}_{1} \cap \mathcal{L}_{2}\right| \geq|\mathbb{K}| \geq 3, \mathcal{L}_{1}$ coincides with $\mathcal{L}_{2}$. This implies that the line $p^{\prime} x_{2}$ intersects $M_{1}$ in a point of $H\left(2 n-1, \mathbb{K}^{\prime}, \theta\right)$. So, $p^{\prime} x_{2}$ is a secant line. But this is impossible: since $p^{\prime} x_{2}$ intersects $M_{2}$ in a point of $H\left(2 n-1, \mathbb{K}^{\prime}, \theta\right)$, it should also be a tangent line.

Now, let $L$ be a secant line through $p^{\prime}$ intersecting $\pi_{i-2}$ in a point not belonging to $H\left(2 n-1, \mathbb{K}^{\prime}, \theta\right)$. We will show that the plane $\left\langle L, p_{i} p\right\rangle$ is non-degenerate. Suppose $p^{*}$ belongs to the radical of $\left\langle L, p_{i} p\right\rangle$. Then the line $p_{i} p^{*}$ is contained in $p_{i}^{\zeta} \cap H\left(2 n-1, \mathbb{K}^{\prime}, \theta\right)$ and intersects $\pi_{i-1}$ in a point of $L \cap H\left(2 n-1, \mathbb{K}^{\prime}, \theta\right) \cap \pi_{i-2}$, a contradiction. Hence, $\left\langle L, p_{i} p\right\rangle$ is non-degenerate.

Let $p_{1}^{\prime}$ and $p_{2}^{\prime}$ be two points of $L \cap H\left(2 n-1, \mathbb{K}^{\prime}, \theta\right)$. Since $\left\langle L, p_{i} p\right\rangle$ is non-degenerate, $\left\langle L, p_{i} p\right\rangle \cap H\left(2 n-1, \mathbb{K}^{\prime}, \theta\right)=\left\langle p_{1}^{\prime}, p_{2}^{\prime}, p_{i}\right\rangle_{\mathcal{N}}\left(\right.$ recall Lemma 3.1). Since $\pi_{i-1} \cap H\left(2 n-1, \mathbb{K}^{\prime}, \theta\right)=$ $\left\langle p_{1}, p_{2}, \ldots, p_{i-1}\right\rangle_{\mathcal{N}}$ (induction hypothesis), $\left\langle p_{1}^{\prime}, p_{2}^{\prime}, p_{i}\right\rangle_{\mathcal{N}} \subseteq\left\langle p_{1}, p_{2}, \ldots, p_{i}\right\rangle_{\mathcal{N}}$. It follows that $p \in\left\langle p_{1}, p_{2}, \ldots, p_{i}\right\rangle_{\mathcal{N}}$. This proves that every point of $\left(\pi_{i} \cap H\left(2 n-1, \mathbb{K}^{\prime}, \theta\right)\right) \backslash\left(p_{i}^{\zeta} \cap \pi_{i}\right)$ belongs to $\left\langle p_{1}, p_{2}, \ldots, p_{i}\right\rangle_{\mathcal{N}}$.

(II) Let $p$ denote an arbitrary point of $\left(p_{i}^{\zeta} \cap \pi_{i}\right) \cap H\left(2 n-1, \mathbb{K}^{\prime}, \theta\right)$ and let $L$ denote an arbitrary line of $\pi_{i}$ through $p$ not contained in $\left(p^{\zeta} \cap \pi_{i}\right) \cup\left(p_{i}^{\zeta} \cap \pi_{i}\right)$. Then $L$ is a secant line. Since $\left(L \cap H\left(2 n-1, \mathbb{K}^{\prime}, \theta\right)\right) \backslash\{p\} \subseteq\left\langle p_{1}, \ldots, p_{i}\right\rangle_{\mathcal{N}}$, also $p \in\left\langle p_{1}, \ldots, p_{i}\right\rangle_{\mathcal{N}}$.

By (I) and (II), every point of $\pi_{i} \cap H\left(2 n-1, \mathbb{K}^{\prime}, \theta\right)$ belongs to $\left\langle p_{1}, p_{2}, \ldots, p_{i}\right\rangle_{\mathcal{N}}$, i.e. $\left\langle p_{1}, p_{2}, \ldots, p_{i}\right\rangle_{\mathcal{N}}=\pi_{i} \cap H\left(2 n-1, \mathbb{K}^{\prime}, \theta\right)$.

Remark. In the finite case, it is possible to give a much shorter proof of Lemma 3.3 due to the nonexistence of exterior lines.

\subsection{A sequence of numbers}

For every $n \in \mathbb{N} \backslash\{0,1\}$ and every $j \in\{0, \ldots, n\}$, we now define a number $f(n, j)$. For $n=2$, we define

$$
f(2,0)=f(2,1)=f(2,2)=2 .
$$

Suppose that for some $n \geq 2$, we have defined $f(n, j)$ for all $j \in\{0, \ldots, n\}$. Then we define

$$
\begin{aligned}
\lambda(n) & :=\sum_{j=0}^{n} f(n, j), \\
f(n+1,0) & :=\lambda(n), \\
f(n+1,1) & :=\lambda(n), \\
f(n+1,2) & :=\lambda(n), \\
f(n+1, k) & :=\sum_{j=k-1}^{n} f(n, j) \text { for every } k \in\{3, \ldots, n+1\} .
\end{aligned}
$$


The numbers $f(n, j), \lambda(n)$ were defined by Cooperstein in [6]. He also proved the following.

Lemma 3.4 ([6, Lemma 4.2]) Let $n \geq 2$. Then

$$
\begin{aligned}
f(n, 0) & =\left(\begin{array}{c}
2 n-2 \\
n-1
\end{array}\right), \\
f(n, 1) & =\left(\begin{array}{c}
2 n-2 \\
n-1
\end{array}\right), \\
f(n, j) & =2\left(\begin{array}{c}
2 n-1-j \\
n-j
\end{array}\right) \text { for every } j \in\{2, \ldots, n\}, \\
\lambda(n) & =\left(\begin{array}{c}
2 n \\
n
\end{array}\right) .
\end{aligned}
$$

\subsection{A generating set of $D H\left(2 n-1, \mathbb{K}^{\prime}, \theta\right),|\mathbb{K}| \neq 2$}

Let $\Delta$ be the dual polar space associated with a nonsingular $\theta$-Hermitian variety $H(2 n-$ $\left.1, \mathbb{K}^{\prime}, \theta\right)$ of Witt-index $n \geq 2$ in $\mathrm{PG}\left(2 n-1, \mathbb{K}^{\prime}\right)$. Let $\zeta$ denote the Hermitian polarity of $\mathrm{PG}\left(2 n-1, \mathbb{K}^{\prime}\right)$ associated with $H\left(2 n-1, \mathbb{K}^{\prime}, \theta\right)$. The maximal subspaces of $H\left(2 n-1, \mathbb{K}^{\prime}, \theta\right)$ through a given point $x$ of $H\left(2 n-1, \mathbb{K}^{\prime}, \theta\right)$ determine a max $M(x)$ of $\Delta$. The discussion in this subsection is based on Cooperstein [6].

The proof of the following lemma is completely similar to the proofs of Lemmas 2.4 and 2.5 and hence we omit it.

Lemma 3.5 ( $i)$ Suppose $x, y$ are non-orthogonal points of $H\left(2 n-1, \mathbb{K}^{\prime}, \theta\right)$ and put $L:=$ $x y \cap H\left(2 n-1, \mathbb{K}^{\prime}, \theta\right)$. Then $\langle M(x), M(y)\rangle_{\Delta}=\bigcup_{z \in L} M(z)$.

(ii) Suppose $x$ and $y$ are distinct orthogonal points of $H\left(2 n-1, \mathbb{K}^{\prime}, \theta\right)$. Then $\langle M(x)$, $M(y)\rangle_{\Delta}=M(x) \cup M(y)$.

Lemma 3.6 Suppose $\mathbb{K}$ is not isomorphic to $\mathbb{F}_{2}$. Let $p_{1}, \ldots, p_{2 n}$ be points of $H(2 n-$ $\left.1, \mathbb{K}^{\prime}, \theta\right)$ satisfying the properties (1) - (3) of Lemma 3.2. Then $\left\langle M\left(p_{1}\right), M\left(p_{2}\right), \ldots\right.$, $\left.M\left(p_{n+1}\right)\right\rangle_{\Delta}$ coincides with the whole point-set of $\Delta$.

Proof. Put $C=\left\langle p_{1}, \ldots, p_{n+1}\right\rangle$. Then $\operatorname{dim}(C)=n$. By Lemmas 3.3 and 3.5,

$$
\left\langle M\left(p_{1}\right), M\left(p_{2}\right), \ldots, M\left(p_{n+1}\right)\right\rangle_{\Delta}=\bigcup_{z \in C} M(z) .
$$

If $x$ is a point of $\Delta$ (i.e., an $(n-1)$-dimensional subspace contained in $H\left(2 n-1, \mathbb{K}^{\prime}, \theta\right)$ ), then $\operatorname{dim}(x \cap C) \geq 0$ since $\operatorname{dim}(x)=n-1$ and $\operatorname{dim}(C)=n$. Hence, $x \in \bigcup_{z \in C} M(z)=$ $\left\langle M\left(p_{1}\right), M\left(p_{2}\right), \ldots, M\left(p_{n+1}\right)\right\rangle_{\Delta}$. This proves the lemma.

Lemma 3.7 Suppose $\mathbb{K}$ is not isomorphic to $\mathbb{F}_{2}$. Let $p_{1}, p_{2}, \ldots, p_{2 n}$ be 2 n points satisfying the conditions (1) - (3) of Lemma 3.2. Put $B_{0}=\emptyset$ and $B_{j}=\left\langle M\left(p_{1}\right), \ldots, M\left(p_{j}\right)\right\rangle_{\Delta}$ for every $j \in\{1, \ldots, n\}$. Then for every $j \in\{0, \ldots, n\}$, there exists a set $X$ of points in $M\left(p_{j+1}\right)$ satisfying: 
(i) $|X|=f(n, j)$;

(ii) $\left\langle\left(B_{j} \cap M\left(p_{j+1}\right)\right) \cup X\right\rangle_{\Delta}=M\left(p_{j+1}\right)$.

Proof. We will prove the lemma by induction on $n$.

Suppose $n=2$ and $j \in\{0,1,2\}$. Then there exists a set $X$ of size $f(2, j)=2$ such that $\langle X\rangle_{\Delta}=M\left(p_{j+1}\right)$. Hence, also $\left\langle\left(B_{j} \cap M\left(p_{j+1}\right)\right) \cup X\right\rangle_{\Delta}=M\left(p_{j+1}\right)$.

Suppose that $n \geq 3$ and that the lemma holds for smaller values of $n$. By the induction hypothesis and Lemma 3.6, every $M\left(p_{i}\right), i \in\{1, \ldots, 2 n\}$, can be generated by $\lambda(n-1)=$ $\sum_{i=0}^{n-1} f(n-1, i)$ points. As a consequence, the claim holds if $j \in\{0,1,2\}$. So, suppose $j \geq 3$. The singular subspaces through $p_{j+1}$ define a polar space of type $H\left(2 n-3, \mathbb{K}^{\prime}, \theta\right)$ which lives in the projective space $p_{j+1}^{\zeta} / p_{j+1}$. Let $D H\left(2 n-3, \mathbb{K}^{\prime}, \theta\right)$ denote the dual polar space associated with this polar space. There exists a natural bijective correspondence between the points of $D H\left(2 n-3, \mathbb{K}^{\prime}, \theta\right)$ and the points of the max $M\left(p_{j+1}\right)$. Now, let $\bar{g}_{i}$, $i \in\{1, \ldots, 2 n\}$, be a nonzero vector of $V$ such that $p_{i}=\left\langle\bar{g}_{i}\right\rangle$. Put

- $p_{i}^{\prime}=p_{i}=\left\langle\bar{g}_{i}\right\rangle$ for every $i \in\{1, \ldots, j-1\}$,

- $p_{j}^{\prime}=\left\langle\left(\bar{g}_{j+2}, \bar{g}_{j+1}\right) \bar{g}_{j}-\left(\bar{g}_{j}, \bar{g}_{j+1}\right) \bar{g}_{j+2}\right\rangle$,

- $p_{i}^{\prime}=p_{i+2}=\left\langle\bar{g}_{i+2}\right\rangle$ for every $i \in\{j+1, \ldots, 2 n-2\}$.

Notice that all these points belong to $H\left(2 n-1, \mathbb{K}^{\prime}, \theta\right)$ and to $p_{j+1}^{\zeta}$. Put $p_{i}^{\prime \prime}=p_{i}^{\prime} p_{j+1}$ for every $i \in\{1, \ldots, 2 n-2\}$. Then $p_{i}^{\prime \prime}, i \in\{1, \ldots, 2 n-2\}$, are points of $H\left(2 n-3, \mathbb{K}^{\prime}, \theta\right)$ satisfying properties (1), (2) and (3) of Lemma 3.2. Let $M^{\prime}\left(p_{i}^{\prime \prime}\right), i \in\{1, \ldots, 2 n-2\}$, denote the max of $M\left(p_{j+1}\right)$ consisting of all maximal singular subspaces through $p_{i}^{\prime} p_{j+1}$. Then $M^{\prime}\left(p_{i}^{\prime \prime}\right)=M\left(p_{i}^{\prime}\right) \cap M\left(p_{j+1}\right)$. The subspace $B_{j} \cap M\left(p_{j+1}\right)$ of $M\left(p_{j+1}\right)$ contains the maxes $M\left(p_{i}\right) \cap M\left(p_{j+1}\right)=M\left(p_{i}^{\prime}\right) \cap M\left(p_{j+1}\right)=M^{\prime}\left(p_{i}^{\prime \prime}\right), i \in\{1, \ldots, j-1\}$. By Lemma 3.6 and the induction hypothesis applied to the maxes $M^{\prime}\left(p_{i}^{\prime \prime}\right), i \in\{1, \ldots, 2 n-2\}$, of $M\left(p_{j+1}\right)$, we see that there exists a set $X$ of size $f(n-1, j-1)+\cdots+f(n-1, n-1)=f(n, j)$ such that $\left\langle\left(B_{j} \cap M\left(p_{j+1}\right)\right) \cup X\right\rangle_{\Delta}=M\left(p_{j+1}\right)$. This proves the lemma.

The following corollary is precisely Theorem 1.3.

Corollary 3.8 If $\mathbb{K} \neq \mathbb{F}_{2}$, then the dual polar space $D H\left(2 n-1, \mathbb{K}^{\prime}, \theta\right)$ can be generated by $\sum_{j=0}^{n} f(n, j)=\lambda(n)=\left(\begin{array}{c}2 n \\ n\end{array}\right)$ points.

Proof. By Lemmas 3.6 and 3.7.

\section{References}

[1] R. J. Blok. The generating rank of the symplectic grassmannians: hyperbolic and isotropic geometry. European J. Combin. 28 (2007), 1368-1394.

[2] A. Blokhuis and A. E. Brouwer. The universal embedding dimension of the binary symplectic dual polar space. Discrete Math. 264 (2003), 3-11. 
[3] W. Burau. Mehrdimensionale projecktive und Högere Geometrie, Berlin, 1961.

[4] P. J. Cameron. Dual polar spaces. Geom. Dedicata 12 (1982), 75-85.

[5] B. N. Cooperstein. On the generation of some dual polar spaces of symplectic type over GF(2). European J. Combin. 18 (1997), 741-749.

[6] B. N. Cooperstein. On the generation of dual polar spaces of unitary type over finite fields. European J. Combin. 18 (1997), 849-856.

[7] B. N. Cooperstein. On the generation of dual polar spaces of symplectic type over finite fields. J. Combin. Theory Ser. A 83 (1998), 221-232.

[8] B. N. Cooperstein. On the generation of some embeddable GF(2) geometries. J. Algebraic Combin. 13 (2001), 15-28.

[9] B. N. Cooperstein. Generation of embeddable incidence geometries: a survey. Quad. Mat. 12 (2003), 29-57.

[10] B. De Bruyn. Near polygons. Birkhäuser, Basel, 2006.

[11] B. De Bruyn. A decomposition of the natural embedding spaces for the symplectic dual polar spaces. Linear Algebra and its Applications, to appear.

[12] B. De Bruyn. On the Grassmann-embeddings of the Hermitian dual polar spaces. Linear and Multilinear Algebra, to appear.

[13] W. M. Kantor. Subgroups of classical groups generated by long root elements. Trans. Amer. Math. Soc. 248 (1979), 347-379.

[14] A. Kasikova and E. E. Shult. Absolute embeddings of point-line geometries. J. Algebra 238 (2001), 265-291.

[15] P. Li. On the universal embedding of the $S p_{2 n}(2)$ dual polar space. J. Combin. Theory Ser. A 94 (2001), 100-117.

[16] P. Li. On the universal embedding of the $U_{2 n}(2)$ dual polar space. J. Combin. Theory Ser. A 98 (2002), 235-252.

[17] M. A. Ronan. Embeddings and hyperplanes of discrete geometries. European J. Combin. 8 (1987), 179-185.

[18] E. E. Shult and A. Yanushka. Near n-gons and line systems. Geom. Dedicata 9 (1980), $1-72$.

[19] J. Tits. Buildings of Spherical Type and Finite BN-pairs. Lecture Notes in Mathematics 386. Springer, Berlin, 1974. 